\title{
Incidência de obesidade em gestantes diabéticas com ou sem o uso de terapêutica
}

\section{medicamentosa}

\author{
Obesity incidence in diabetic pregnant women with or without use of drug therapy \\ Incidencia de obesidad en mujeres diabéticas embarazadas con o sin uso de terapia medicamentosa
}

Recebido: 10/06/2021 | Revisado: 18/06/2021 | Aceito: 28/06/2021 | Publicado: 11/07/2021

Isabela Rezende

ORCID: https://orcid.org/0000-0002-0981-1286 Centro Universitário da Fundação Assis Gurgacz, Brasil

E-mail: isabelarfarias@gmail.com

Adriano Luiz Possobon

ORCID: https://orcid.org/0000-0001-9720-2482 Centro Universitário da Fundação Assis Gurgacz, Brasil

E-mail: possobon@msn.com

Letícia Frare

ORCID: https://orcid.org/0000-0002-8072-9410 Centro Universitário da Fundação Assis Gurgacz, Brasil

E-mail: leticia.zfrare@gmail.com

Eron Celant Espíndola

ORCID: https://orcid.org/0000-0001-7698-9108 Centro Universitário da Fundação Assis Gurgacz, Brasil E-mail: eroncelant@gmail.com

Giuliana Medeiros

ORCID: https://orcid.org/0000-0003-1953-8153 Centro Universitário da Fundação Assis Gurgacz, Brasil E-mail: giummoreira05@gmail.com

\begin{abstract}
Resumo
Neste trabalho será estudada a prevalência de obesidade entre dois grupos de gestantes com diabetes, separando entre aquelas que necessitam de terapêutica medicamentosa e as que conseguem bom controle com manejo não medicamentoso. O diabetes gestacional é a intolerância aos carboidratos diagnosticada pela primeira vez durante a gestação. É o problema metabólico mais comum durante esse período, sendo a obesidade um dos principais fatores de risco para o desenvolvimento dessa patologia, a qual gera inúmeros malefícios tanto para a paciente quanto para o concepto. Quando o diabetes é diagnosticado, podendo ser através dos números da glicemia em jejum ou através do exame TOTG 75g, pode-se obter um desfecho gestacional desfavorável, necessitando o mais breve possível da regulação da glicemia sanguínea. Para isso, diferentes estratégias podem ser empregadas, como a não medicamentosa, por meio do exercício físico e da alimentação balanceada, e a medicamentosa com o uso dos hipoglicemiantes orais ou da insulina.
\end{abstract}

Palavras-chave: Diabetes gestacional; Obesidade; Insulina; Hipoglicemiantes; Dieta.

\begin{abstract}
In this work, the prevalence of obesity among two groups of pregnant women with diabetes will be studied, separating between those who need drug therapy and those who achieve good control with non-drug management. Gestational diabetes is the intolerance to carbohydrates first diagnosed during pregnancy. It is the most common metabolic problem during this period. Being, obesity one of the main risk factors for the development of this pathology, which generates innumerable harms for both the patient and the fetus. When diabetes is diagnosed, may be through fasting glucose numbers or by $75 \mathrm{~g}$ OGTT test, one can obtain an adverse pregnancy outcome, requiring blood glucose regulation. For this, different strategies can be employed such as non-medication with physical exercise and balanced diet or medication with the use of oral hypoglycemic agents or insulin.
\end{abstract}

Keywords: Gestational diabetes; Obesity; Insulin; Hypoglycemic agents; Diet.

\section{Resumen}

En este trabajo se estudiará la prevalencia de obesidad entre dos grupos de mujeres embarazadas con diabetes, separando entre aquellas que necesitan terapia con medicamentos y aquellas que tienen buen control con un manejo no farmacológico. El diabetes gestacional es la intolerancia a los carbohidratos que se diagnostica por primera vez durante el embarazo. Es el problema metabólico más común durante este período. La obesidad es uno de los principales factores de riesgo para el desarrollo de esta patología, que genera innumerables daños tanto para el paciente como para el feto. 
Cuando se diagnostica diabetes, a través de los niveles de glucosa en sangre en ayunas o mediante el examen TOTG $75 \mathrm{~g}$, se puede obtener un resultado gestacional desfavorable, requiriendo lo antes posible la regulación de glucosa en sangre. Para ello, se pueden utilizar diferentes estrategias, como no medicamentosa, a través del ejercicio y dieta equilibrada o medicación con el uso de hipoglucemiantes orales o insulina.

Palabras clave: Diabetes gestacional; Obesidad; Insulina; Hipoglucemiantes; Dieta.

\section{Introdução}

A obesidade possui etiologia multifatorial e complexa. Ela está relacionada com a predisposição genética, com as mudanças fisiológicas do metabolismo do corpo, com padrões culturais e questões socioeconômicas (Alves et al., 2019). A prevalência de obesidade praticamente dobrou nos últimos 20 anos. Em países do ocidente, seus índices em gestantes chegam a quase $30 \%$, e estima-se que mais de $40 \%$ destas ganhem peso acima da faixa recomendada para seu índice de massa corporal (IMC), segundo o Institute of Medicine (IOM). Assim, mulheres obesas apresentam risco maior de complicações na gravidez, incluindo as síndromes hipertensivas da gestação e, o alvo do nosso estudo, o diabetes mellitus gestacional (DMG) (Ferreira et al., 2019; Coustan, 2013; Alfadhli, 2015).

O diabetes mellitus (DM) é uma síndrome clínica que tem como característica a hiperglicemia decorrente de uma deficiência na efetividade da insulina ou de uma diminuição desta, causando distúrbios metabólicos de macro nutrientes (carboidratos, lipídeos e proteínas), água e eletrólitos. É uma patologia que se caracteriza por uma disfunção pancreática, em que este órgão pode sofrer desde um funcionamento inadequado até a perda total da sua capacidade de produção do hormônio insulina (Menicatti; Fregonesi, 2006; Spaight et al, 2016; Chen et al, 2015; Cheng; Caughey, 2008).

É uma intolerância à glicose de graus variáveis com início ou primeiro diagnóstico durante a gestação (Maganha et al, 2004). Essa alteração metabólica tem uma prevalência entre 3\% a 25\%. Na última década tem aumentado progressivamente o número de mulheres diagnosticadas com essa patologia em decorrência, entre outras coisas, do aumento da idade materna, da piora dos hábitos de vida e do crescente número de mulheres obesas (Alves et al., 2019). O tratamento deve visar a diminuição dos índices glicêmicos sanguíneos buscando reduzir os danos causados à saúde da mãe e do concepto. Inicialmente, o recomendado é a mudança de hábitos de vida, tais como dieta balanceada e prática de exercícios físicos. Quando tais medidas não são satisfatoriamente suficientes para alcançar níveis desejados de glicose, é necessário que seja adotado o tratamento farmacológico. Esse tipo de tratamento pode ser realizado com o uso de hipoglicemiantes orais e/ou da insulinoterapia. Acreditase que de $15 \%$ a $60 \%$ das pacientes necessitem do uso da farmacologia em conjunto com a mudança de hábitos para que haja o controle da doença (Souza et al., 2019; Lende; Rijhsinghani, 2020; Zheng et al, 2017; Di Biase et al, 2019; Koning et al, 2016).

A gestação por ser um estado hiperinsulinêmico do organismo é caracterizada por uma diminuição da sensibilidade à insulina, parcialmente explicada pela presença de hormônios diabetogênicos, como a progesterona, o cortisol, a prolactina e o hormônio lactogênico placentário. Assim, os níveis glicêmicos de jejum tendem a ser mais baixos na gestante, todavia, os valores pós-prandiais tendem a ser mais altos, principalmente naquelas em que não há aumento adequado da liberação de insulina. Os picos pós-prandiais podem ser explicados pois pacientes com DMG apresentam uma diminuição acentuada da sensibilidade periférica à insulina, como no diabetes tipo 2, além de uma secreção diminuída desse hormônio (Maganha et al, 2004). Contudo, no primeiro trimestre tais alterações têm pouco efeito diretamente sobre o metabolismo dos carboidratos. E conforme ocorre o crescimento placentário há um aumento na produção de hormônios que antagonizam a ação da insulina. Devido a esses fatores, no segundo e terceiro trimestres, é característico que haja uma maior resistência à insulina, aumentando sua concentração no organismo da gestante. Assim, pode ocorrer a instalação do diabetes mellitus gestacional (DMG) que se define quando a demanda da produção de insulina é maior que a capacidade das células $\beta$ pancreáticas em produzi-la (Menicatti; Fregonesi, 2006; Baz et al, 2016). 
Atualmente são utilizados diferentes métodos para o diagnóstico de DMG. A International Association of the Diabetes and Pregnancy Study Groups (IADPSG) decidiu, em 2010, basear os critérios diagnósticos nos resultados do estudo Hyperglycemia and Adverse Pregnancy Outcomes (HAPO), uma pesquisa que visou encontrar um ponto de corte que ligasse a hiperglicemia materna a eventos perinatais adversos. Foram propostos, então, novos valores de referência para o a glicemia em jejum, na primeira e segunda hora, sendo: $\geq 92 \mathrm{mg} / \mathrm{dL}, \geq 180 \mathrm{mg} / \mathrm{dL}$ e $\geq 153 \mathrm{mg} / \mathrm{dL}$, respectivamente. E quando um valor fosse anormal já seria diagnosticado o DMG. A OMS, em 2013, também adotou o uso desses valores para o diagnóstico de DMG, destacando que um valor de glicemia de jejum $\geq 126 \mathrm{mg} / \mathrm{dL}$ ou após sobrecarga $>200 \mathrm{mg} / \mathrm{dL}$ seriam critérios para fechar diagnóstico de diabetes mellitus franco, e não DMG. (Sociedade Brasileira de Diabetes, 2020).

Embora um consenso entre as sociedades médicas internacionais ainda não tenha sido alcançado, em 2017, a Sociedade Brasileira de Diabetes (SBD), a Federação Brasileira das Associações de Ginecologia e Obstetrícia (FEBRASGO), a Organização Pan-Americana de Saúde (OPAS) e o Ministério da Saúde do Brasil uniram-se a fim de definirem uma padronização do rastreamento e diagnóstico do DMG no país. Sendo assim, os critérios normatizados pela Sociedade Brasileira de Diabetes (2020), foram adaptados dos critérios aceitos pela OMS em 2013. E, também levaram em consideração aspectos importantes como a viabilidade financeira e a disponibilidade técnica. Dessa maneira, definiu-se que a investigação de DMG deve ser feita em todas as gestantes que não possuam diagnóstico prévio de diabetes. Na primeira consulta pré-natal, deve ser solicitada glicemia de jejum, objetivando a detecção de diabetes preexistente. Um valor de hiperglicemia detectada em qualquer período gestacional pode ser diferenciado em DM diagnosticado na gestação ou em DMG. Assim, o rastreamento pode ser dividido em: (1) Gestantes com início do pré-natal <20 semanas, deve-se fazer a investigação imediata da glicemia de jejum. Se o valor encontrado for $\geq 126 \mathrm{mg} / \mathrm{dL}$, será feito o diagnóstico de DM diagnosticado na gestação. Se a glicemia em jejum $\geq 92 \mathrm{mg} / \mathrm{dL}$ e $<$ $126 \mathrm{mg} / \mathrm{dL}$, o dado será de DMG. Em ambos os casos, o resultado deve ser confirmado com uma segunda dosagem da glicemia de jejum. Se a Glicemia de Jejum (GJ) for $<92 \mathrm{mg} / \mathrm{dl}$ será realizado o TOTG 75g entre a 24 e 28 semana. (2) Se início do prénatal entre 20-28 semanas, realiza-se o TOTG 75g entre a 24 e 28 semana de gestação. (3) Quando pré-natal iniciado após 28 semana, o TOTG 75g é realizado imediatamente. O diagnóstico é definido quando dosagem em jejum: 92 a $125 \mathrm{mg} / \mathrm{dl} ; 1$ hora após: > $180 \mathrm{mg} / \mathrm{dl}$; e 2 horas após: 153 a $199 \mathrm{mg} / \mathrm{dl}$.

Assim como outros tipos de diabetes, o tratamento do DMG deve incluir uma dieta balanceada e individualizada e a prática de exercícios físicos e, quando há indicação, inclui-se o uso de fármacos. Nas primeiras etapas do tratamento prescrevese dieta e atividade física e, se as metas glicêmicas preconizadas não forem atingidas em duas semanas, o tratamento farmacológico é implantado (Weinert et al., 2011). A terapia medicamentosa padrão para o diabetes gestacional é a insulinoterapia subcutânea. Entretanto, não é um tratamento muito aceito pelas pacientes devido a sua complexidade de administração e ao ganho indesejado de peso. Como alternativa terapêutica, os hipoglicemiantes orais são igualmente efetivos quando comparados a insulinoterapia, no tratamento do diabetes gestacional (Weinert et al., 2011).

O tratamento inicial do DMG consiste na orientação alimentar que auxilie o ganho de peso adequado e o controle metabólico. Assim, a terapia nutricional visa evitar o ganho excessivo de peso pelas gestantes, além de gerar menor taxa de macrossomia fetal e de complicações perinatais. O cálculo do valor calórico total da dieta pode ser feito de acordo com o índice de massa corporal (IMC) e visa que a gestante tenha um ganho de peso em média de $300 \mathrm{~g}$ a $400 \mathrm{~g}$ por semana, a partir do segundo trimestre de gravidez. O recomendado pela SBD é que, o valor calórico total prescrito deve ter $40 \%$ a $45 \%$ de carboidratos, $15 \%$ a $20 \%$ de proteínas e 30\% a $40 \%$ de gorduras (Sociedade Brasileira de Diabetes, 2015). Dietas muito restritivas (com menos de $1.500 \mathrm{kcal} /$ dia) podem induzir cetonemia e não são recomendadas. Já, dietas com restrição calórica moderada (1.600 - $1.800 \mathrm{kcal})$ não acarretam cetose e são efetivas no controle do ganho de peso materno e no controle dos níveis glicêmicos, sendo mais recomendadas (Weinert et al., 2011). 
A prática de exercícios na gestação tem como benefício a redução da glicemia, a redução do ganho excessivo de peso materno e a diminuição da incidência de macrossomia fetal. Dessa forma, deve ser recomendada para todas as gestantes diabéticas, na ausência de contraindicações, realizar 15 a 30 minutos de atividade física diária, como caminhadas ou exercícios de baixo impacto. Deve ser 8 realizada a monitorização da atividade fetal e da glicemia capilar antes e após a atividade, para que não haja prejuízo no decorrer do exercício (Weinert et al., 2011).

Quando um ou mais valores de glicemia capilar estiverem além dos alvos terapêuticos (jejum < $95 \mathrm{mg} / \mathrm{dL}, 1 \mathrm{~h}<140$ $\mathrm{mg} / \mathrm{dL}$ e $2 \mathrm{~h}<120 \mathrm{mg} / \mathrm{dL}$ ) após duas semanas com medidas de mudança de estilo de vida, ou quando a avaliação ecográfica mostrar sinais de crescimento fetal excessivo, indica-se instituição do tratamento farmacológico. As diretrizes, tanto nacionais quanto internacionais, divergem sobre o tratamento medicamentoso do diabetes gestacional (Sociedade Brasileira de Diabetes, 2020). A SBD sugere o uso de insulina como tratamento padrão, enquanto o National Institute for Health and Clinical Excellence (NICE) recomenda o uso de metformina e a International Diabetes Federation (IDF) aponta a metformina e a glibenclamida como opções de tratamento, principalmente em situações em que o uso de insulina é difícil (Weinert et al., 2011). A dose inicial de insulina deve ser em torno de 0,5 U/kg, com ajustes individualizados para cada caso. Em geral, associam-se insulinas humanas de ações intermediária e rápida. Os análogos de insulina asparte e lispro têm vantagens sobre a insulina regular, promovendo melhor controle dos níveis de glicemia pós-prandiais com menor ocorrência de hipoglicemias. Acredita-se que de $15 \%$ a $60 \%$ das pacientes necessitem do uso da farmacologia em conjunto com a mudança de hábitos para que haja o controle da doença (Souza et al., 2019).

A Organização Mundial da Saúde (OMS) considera a obesidade como uma epidemia mundial e um dos maiores desafios da saúde pública do século 21. A obesidade tem uma causa multifatorial e complexa, podendo estar relacionada com a predisposição genética, com mudanças fisiológicas do sistema endócrino, com o histórico familiar de obesidade, com crenças culturais, além de problemas socioeconômicos (Alves et al., 2019). Diante disso, a Sociedade Brasileira de Diabetes (2020), afirma que a incidência de DMG tem aumentado na mesma velocidade do aumento do DM2 e da obesidade na população feminina do mundo todo. A obesidade na gravidez é definida como um índice de massa corporal (IMC) maior ou igual a $30 \mathrm{~kg} / \mathrm{m} 2$ na primeira consulta pré-natal. Essa classificação, pode ser subdividida em: classe I (30,0 - 34,9 kg/m2), classe II (35,0-39,9 kg/m2) e classe III $(40 \mathrm{~kg} / \mathrm{m} 2)$. Sendo que quanto maior a classe que a gestante se enquadra, maiores são os riscos de complicações durante a gestação. Nesse sentido, o trabalho de Julia et al (2017) revelou que mães de RN macrossômicos pesavam antes de engravidar, em média, $6 \mathrm{~kg}$ a mais do que as mães dos normossômicos. Em relação ao ganho de peso durante a gestação, as primeiras ganharam, em torno de 3,5 kg mais do que as segundas. Assim, a diabetes materna, o peso prévio à gravidez e seu IMC mostraram ser fatores de risco para macrossomia neonatal, além de poder predispor a hipoglicemia nas primeiras 72 horas de vida do recém-nascido.

Assim, essa pesquisa tem como base a relação entre o diabetes gestacional, o IMC dessas gestantes e o tipo do tratamento instituído para o controle da desordem metabólica, visando manter um índice glicêmico satisfatoriamente adequado a fim de reduzir os danos causados à saúde da mãe e do concepto. Uma vez que essa patologia se mostrou, ao longo dos anos, uma causa importante de prejuízos aos desfechos gestacionais.

\section{Metodologia}

Caracteriza-se por um estudo retrospectivo, quantitativo, de caráter descritivo realizado de maneira transversal. Foi desenvolvido no Centro de Atendimento Especializado (CAE) em Cascavel - PR, no ano de 2020. Nesta pesquisa a população se constituiu por gestantes com diagnóstico de diabetes mellitus gestacional e que realizaram acompanhamento entre junho de 2019 e junho de 2020. Por se tratar de uma pesquisa com seres humanos o seguinte estudo está em cumprimento com a Resolução 466/2012 do Conselho Nacional de Saúde. Como base metodológica utilizou-se o trabalho de Pereira et al (2018). 
O instrumento dessa coleta se baseou na revisão de prontuários das gestantes com diabetes mellitus gestacional confirmado através da glicemia de jejum do primeiro trimestre ou do exame de TOTG 75g do segundo trimestre. Para obtenção dos resultados foram utilizados os prontuários que se encontram no Centro de Atendimento Especializado e, então, os dados foram comparados com os existentes na literatura. Foram excluídos da coleta de dados prontuários que não apresentaram todas as informações pesquisadas ou que apresentaram equívoco no diagnóstico.

A partir da análise dos prontuários, foram coletadas informações tais como qual exame foi utilizado para o auxílio do diagnóstico do DMG, o IMC e qual o tratamento está sendo empregado para o controle da patologia.

Os dados computados foram submetidos ao teste de hipóteses utilizando o software RStudio na versão 1.3.959. Foi realizado o teste de comparação de médias T-Student quando foram cumpridos os requisitos para realização deste teste (como normalidade de distribuição entre as amostras coletadas com homogeneidade de variâncias). Quando algum requisito não foi cumprido optou-se pelo teste de Mann-Whitney por tratar-se de comparações envolvendo amostras independentes. Após serem analisados os dados foram distribuídos em tabelas para melhor compreensão dos resultados.

\section{Resultados e Discussão}

Foram analisados 103 prontuários, de pacientes atendidas entre junho de 2019 e junho de 2020, no CAE (Centro da Atenção Especializada) em Cascavel-PR, sendo estes todos de mulheres gestantes diagnosticadas com diabetes durante o período gestacional atual. Dentro dessa amostra 11 pacientes (11\%) tinham DM/DMG prévio à gestação atual e 92 pacientes $(89 \%)$ receberam o diagnóstico de DMG durante a gestação vigente (Tabela 1).

Entre as pacientes diagnosticadas com DMG, 54 gestantes (52\%) receberam seu diagnóstico através da glicemia de jejum, coletada nos exames de rotina do primeiro trimestre. E as demais, 38 gestantes (37\%) foram diagnosticadas pelo Teste Oral de Tolerância a Glicose 75g (TOTG) (Gráfico 1).

Gráfico 1: Distribuição dos subtipos de diagnósticos de Diabetes em Gestantes atendidas em um centro de atenção especializada em Cascavel/PR entre 2019-2020 (n=103).

\section{GESTANTES}

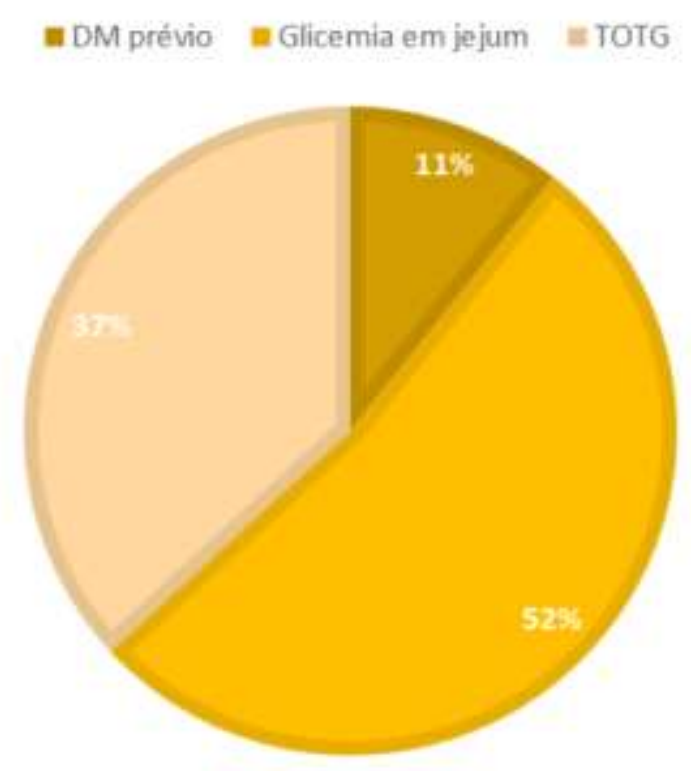

Fonte: Autores (2021). 
O estudo analisou que a mediana da glicemia das pacientes no exame indicado para diagnóstico do DMG - no primeiro trimestre - ficou entre 98mg/dl no teste da glicemia em jejum e 152mg/dl 1 hora após TOTG 75g e 127mg/dl 2 horas após TOTG $75 \mathrm{~g}$-realizado para diagnóstico no segundo trimestre (Tabela 1).

A amostra coletada mostrou, também, que houve uma variedade entre os IMCs das pacientes estudadas. Uma menor porcentagem das pacientes possuía o IMC na faixa desejável (IMC menor que 25) para uma gestação com menores complicações maternas e fetais, sendo esse valor 7\% (7 pacientes). A maior parte da amostra possuía o IMC maior que 25, o que indica sobrepeso ou diferentes graus de obesidade (Tabela 1).

Assim, 20\% (21 pacientes) tinham o IMC entre 25,01 e 29,99, configurando sobrepeso; $31 \%$ (32 pacientes) entre 30,00 e 34,99, configurando obesidade grau I; $24 \%$ (25 pacientes) entre 35,00 e 39,99, configurando obesidade grau II; e $17 \%$ (18 pacientes) tinham esse índice acima de 40,00, configurando obesidade grau III (Tabela 1).

Ainda, pôde-se ver que dentre as 21 pacientes com IMC na faixa de sobrepeso, 4 tinham diagnóstico de DM prévio, 10 foram diagnosticadas através da glicemia de jejum (primeiro trimestre) e 7 através do TOTG 75g (segundo trimestre). Das 32 pacientes na faixa de obesidade grau I, 3 possuíam DM previamente à atual gestação, 17 foram diagnosticadas pela GJ do primeiro trimestre e 12 pelo TOTG do segundo trimestre. A faixa de obesidade grau II foi encontrada em 25 pacientes da amostra, entre essas nenhuma (0 pacientes) tinham diagnóstico prévio de DM, 15 foram diagnosticadas no primeiro trimestre pela GJ e 10 no segundo pelo TOTG 75g. Ademais, entre as 18 pacientes com o IMC acima de 40, obesidade grau III, 3 tinham DM prévio, 11 tiveram diagnóstico pela GJ no primeiro trimestre e 4 pelo TOTG no segundo trimestre.

Quanto ao tratamento adotado para o controle da doença, a amostra estudada evidenciou que a maior parte das pacientes (67\%) conseguiram sucesso no tratamento apenas utilizando medidas dietéticas, sem a necessidade do uso de medicações. Entretanto, entre as demais pacientes $17 \%$ fizeram uso de metformina, $1 \%$ de glibenclamida e $21 \%$ de insulina (NPH e/ou regular) durante o período gestacional para auxílio no controle da doença (Tabela 1).

Para analisar, entre as pacientes, a terapêutica adotada, dividiu-se as 92 pacientes com diagnóstico de DMG, entre as diagnosticadas no primeiro trimestre (54 gestantes) e as diagnosticadas no segundo trimestre (38 gestantes). Destes dois grupos, separou-se, ainda, diante do tratamento empregado, se a gestante era obesa ou não (Tabela 2).

Dessa maneira, entre as 54 pacientes com diagnóstico pela glicemia de jejum, 39 alcançaram sucesso nas metas glicêmicas a partir do tratamento dietético, sendo que destas 10 não eram obesas e 29 eram. Em meio as 15 que necessitaram de tratamento farmacológico para alcançar as metas glicêmicas, 1 não era obesa e 14 eram (Tabela 2).

Diante das 38 gestantes com diagnóstico pelo TOTG 75g, 30 foram tratadas com mudanças na dieta, destas 11 não eram obesas e 19 eram. E entre aquelas que adotaram o tratamento farmacológico (8 pacientes), 2 não eram obesas e 6 eram (Tabela 2). 
Tabela 1: Informações de mulheres grávidas com Diabetes atendidas em uma unidade de atenção secundária em um município do Oeste do Paraná em 2020 (n=103).

\begin{tabular}{|c|c|c|}
\hline DM Prévio? & $\mathbf{n}$ & Porcentagem \\
\hline Sim & 11 & $11 \%$ \\
\hline Não & 92 & $89 \%$ \\
\hline \multicolumn{3}{|l|}{ IMC } \\
\hline Entre 18,01 e 25,00 & 7 & $7 \%$ \\
\hline Entre 25,01 e 29,99 & 21 & $20 \%$ \\
\hline Entre 30,00 e 34,99 & 32 & $31 \%$ \\
\hline Entre 35,00 e 39,99 & 25 & $24 \%$ \\
\hline Acima de 40,00 & 18 & $17 \%$ \\
\hline \multicolumn{3}{|l|}{ Tratamento Instituído } \\
\hline Apenas alterações na dieta & 69 & $67 \%$ \\
\hline Metformina & 18 & $17 \%$ \\
\hline Glibenclamida & 1 & $1 \%$ \\
\hline Insulina (NPH e/ou regular) & 22 & $21 \%$ \\
\hline \multicolumn{3}{|l|}{ Período do Diagnóstico } \\
\hline Prévio a gestação & 11 & $11 \%$ \\
\hline Primeiro Trimestre & 54 & $52 \%$ \\
\hline Segundo Trimestre & 38 & $37 \%$ \\
\hline \multicolumn{3}{|l|}{ Mediana da Glicemia } \\
\hline Glicemia de Jejum & 98 & $\mathrm{mg} / \mathrm{dL}$ \\
\hline Após 1 hora do TOTG & 152 & $\mathrm{mg} / \mathrm{dL}$ \\
\hline Após 2 horas do TOTG & 127 & $\mathrm{mg} / \mathrm{dL}$ \\
\hline
\end{tabular}

Fonte: Autores (2021)

Tabela 2: Tratamento do diabetes mellitus gestacional por período do diagnóstico em uma unidade de atenção secundária em um município do Oeste do Paraná em 2020 (n=92).

\begin{tabular}{lccc}
\hline Diagnóstico no primeiro trimestre $(\mathbf{n = 5 4})$ & $\mathbf{n}$ & Porcentagem & $\mathbf{p}$ \\
Tratamento apenas com dieta & 39 & & \\
$\quad$ Sem Obesidade & 10 & $26 \%$ & \\
$\quad$ Obesidade & 29 & $74 \%$ & 0,12 \\
Tratamento farmacológico & 15 & & \\
$\quad$ Sem Obesidade & 1 & $7 \%$ & \\
$\quad$ Obesidade & 14 & $93 \%$ & \\
Diagnóstico no segundo trimestre $(\mathbf{n}=\mathbf{3 8})$ & & & \\
Tratamento apenas com dieta & 30 & & \\
$\quad$ Sem Obesidade & 11 & $37 \%$ & \\
$\quad$ Obesidade & 19 & $63 \%$ & \\
Tratamento farmacológico & 8 & & \\
Sem Obesidade & 2 & $25 \%$ & \\
Obesidade & 6 & $75 \%$ & \\
\hline
\end{tabular}

Obs. 1: Não houve associação estatística entre tratamento (apenas com dieta ou farmacológico) com obesidade em gestantes com diagnóstico de Diabetes Mellitus Gestacional no primeiro trimestre $(\mathrm{p}=0,12)$ Obs. 2: Não houve associação estatística entre tratamento (apenas com dieta ou farmacológico) com obesidade em gestantes com diagnóstico de Diabetes Mellitus Gestacional no segundo trimestre $(\mathrm{p}=0,54)$ Fonte: Autores (2021) 
A partir da análise dos dados, o presente estudo demonstrou que as gestantes com DMG que são atendidas no CAE, em sua maioria, apresentam diagnóstico de DMG, obesidade grau I e que fazem tratamento para controle da patologia apenas com mudanças na dieta.

A maior prevalência de gestantes, atendidas nessa instituição, com o diagnóstico no primeiro trimestre através do exame de glicemia em jejum vai em concordância com a literatura, uma vez que nosso estudo analisou que $52 \%$ das gestantes com DMG receberam seu diagnóstico nesse período. E outro estudo, que relaciona o valor da glicemia em jejum do primeiro trimestre e os fatores de risco para gestantes com DMG, indicou que o grupo de gestantes que tinham glicemia de jejum $\geq 85 \mathrm{mg} / \mathrm{dL}$ na primeira consulta, no primeiro trimestre, era formado por 42 gestantes (59,1\% da amostra) (Simon et al, 2013).

Sabe-se que o DMG pode ocorrer em qualquer mulher, entretanto, a partir do estudo em questão, ficou evidente que suas chances são maiores naquelas com IMC maior que 25. Esse fato pode ser devido à resistência insulínica que o corpo desenvolve em pessoas com sobrepeso ou obesas e que durante a gestação, em decorrência da demanda do feto, é aumentada fazendo com que o pâncreas não consiga supri-la (Maganha et al, 2004).

Apesar de estar em concordância quanto ao risco aumentado do desenvolvimento de DMG em pacientes obesas, um estudo recente desenvolvido pelo Serviço de Diabetes do HUPE e apresentado no Congresso Brasileiro de Diabetes despertou atenção para a prevalência significativa de obesas grau III com diabetes gestacional (DMG) que eram acompanhadas pelos Serviços de Obstetrícia e Diabetes da UERJ entre 2011 e 2012. Entre as 43 pacientes estudadas, 58,13\% eram obesas, sendo que em $44 \%$ destas o IMC no momento da concepção era superior a $40 \mathrm{~kg} / \mathrm{m} 2$ (Carneiro et al., 2014). Enquanto nesta pesquisa, a maior prevalência foi em obesas grau I. Além disso, evidenciou-se que grávidas com diagnóstico prévio de DM têm maior prevalência de uso de insulina ( $\mathrm{p}<0.001)$ (Tabela 3) e, também, apresentam menor prevalência do uso isolado da dieta para controlar o diabetes ( $\mathrm{p}$ <0.001) (Tabela 3). Uma das hipóteses para esse resultado é que estas pacientes já apresentavam a doença sob controle com a utilização do fármaco, sendo assim o mesmo tratamento é instituído durante a patologia vigente à gestação. As gestantes com DMG em tratamento apenas com mudanças dietéticas apresentam menores níveis de glicemia de jejum no momento do diagnóstico $(\mathrm{p}<0.001)$ (Tabela 3).

Isso pode ser devido à: (1) Grávidas com a glicemia de jejum mais baixa terem maiores chances de conseguir sucesso no controle glicêmico apenas com medidas dietéticas (Weinert et al., 2011); ou à menor adesão ao tratamento farmacológico, com metformina e/ou insulina pelas outras mulheres. A literatura não descreve relação entre o período do diagnóstico ou o IMC das gestantes e o tipo de tratamento adotado. Desta forma, este estudo pode servir como base para novos trabalhos testarem esta hipótese.

Tabela 3: Correlações estatisticamente significativas $(\mathrm{p}<0,05)$.

\begin{tabular}{lc}
\hline Hipótese Alternativa & Valor p \\
\hline $\begin{array}{l}\text { Grávidas que tiveram DM Prévio têm maior prevalência de uso de insulina na } \\
\text { comparação com outras grávidas com DMG }\end{array}$ & $<0.001$ \\
$\begin{array}{l}\text { Grávidas que tiveram DM Prévio têm menor prevalência de uso apenas de dieta } \\
\text { para controle do diabetes na comparação com outras grávidas com DMG }\end{array}$ & $<0.001$ \\
$\begin{array}{l}\text { Grávidas com DMG em tratamento apenas com dieta têm menor glicemia de } \\
\text { jejum que grávidas utilizando metformina/insulina }\end{array}$ & $<0.001$ \\
$\begin{array}{l}\text { Grávidas com DMG em tratamento com insulina têm maior glicemia de jejum } \\
\text { que grávidas utilizando metformina ou apenas dieta }\end{array}$ & 0.035 \\
$\begin{array}{l}\text { Grávidas com DMG em tratamento com metformina têm maior glicemia de } \\
\text { jejum que grávidas utilizando insulina ou apenas dieta }\end{array}$ & 0.005 \\
\hline
\end{tabular}

Fonte: Autores (2021). 


\section{Conclusão}

Esse trabalho se propôs a analisar o a incidência de obesidade em gestantes diabéticas com ou sem o uso de terapia medicamentosa, no CAE (Centro da atenção especializada) em Cascavel -PR, entre junho de 2019 e junho de 2020.

O estudo em questão analisou 103 prontuários de gestantes atendidas no alto risco. Ao final da pesquisa, pôde-se concluir que a maior parte das pacientes da amostra possuem diagnóstico de diabetes mellitus gestacional, ou seja, não apresentavam a patologia anteriormente e durante o primeiro ou o segundo trimestre tiveram exames laboratoriais que comprovaram o diagnóstico.

Pacientes com diagnóstico prévio persistiram com a desordem durante a gestação vigente. As demais, que eram previamente hígidas, em sua maioria, tiveram o desenvolvimento da doença altamente ligado ao sobrepeso, segundo o resultado da análise dos dados. Dessa maneira, pôde-se comprovar que as gestantes que foram diagnosticadas com DMG, na maior parte da amostra, apresentavam IMC maior que 25 (configurando sobrepeso ou obesidade). Isso deixa claro que esse parâmetro é um fator de risco ao desenvolvimento da patologia.

Ademais, o IMC mais alto também teve relação com o tipo de tratamento adotado para o controle da doença. Diferentes tratamentos foram empregados às gestantes da amostra, variando entre o não farmacológico (mudanças na dieta) e os farmacológicos (hipoglicemiantes orais e/ou insulina). Pacientes com IMC maior ou igual a 30 tiveram mais dificuldade para fazer o controle do diabetes apenas com a mudança na alimentação, necessitando da introdução medicamentosa. As gestantes com DM prévio, estudadas na pesquisa, necessitaram da introdução da insulina para obterem sucesso no controle da patologia. Entretanto, maior parte das gestantes estudadas conseguiu o controle glicêmico apenas com o tratamento dietético.

Dessa forma, ao final desse estudo, conclui-se que o sobrepeso e a obesidade são fatores determinantes no desenvolvimento da patologia e também na terapêutica instituída. O IMC acima de 25 leva ao maior risco de adquirir a desordem metabólica, assim como a maiores chances da necessidade da instituição do tratamento farmacológico.

Futuros artigos podem verificar se as associações apresentadas nesse trabalho observacional são válidas em amostras maiores. Além da adição de mais participantes, podem-se realizar estudos avaliando fatores como escolaridade e situação socioeconômica da gestante para verificar se eles estão associados a necessidade de uso de medicação nos casos de diabetes gestacional.

\section{Referências}

Alves, P., Malheiro, M. F., Gomes, J. C., Ferraz, T., \& Montenegro, N. (2019). Risks of Maternal Obesity in Pregnancy: A Case-control Study in a Portuguese Obstetrical Population. Revista Brasileira de Ginecologia e Obstetricia, 41(12), 682-687. https://doi.org/10.1055/s-0039-3400455

Baz, B., Riveline, J.-P., \& Gautier, J.-F. (2016). Endocrinology Of Pregnancy: Gestational diabetes mellitus: definition, aetiological and clinical aspects. European Journal of Endocrinology, 174(2), R43-R51. https://doi.org/10.1530/EJE-15-0378

Carneiro, J. R. I., Braga, F. O., Cabizuca, C. A., Abi-Abib, R. C., Cobas, R. A., \& Gomes, M. B. (2014). Gestação e obesidade: um problema emergente. Revista Hospital Universitário Pedro Ernesto, 13(3). https://doi.org/10.12957/rhupe.2014.12130

Chen, P., Wang, S., Ji, J., Ge, A., Chen, C., Zhu, Y., Xie, N., \& Wang, Y. (2015). Risk Factors and Management of Gestational Diabetes. Cell Biochemistry and Biophysics, 71(2), 689-694. https://doi.org/10.1007/s12013-014-0248-2

Cheng, Y. W., \& Caughey, A. B. (2008). Gestational diabetes: diagnosis and management. Journal of Perinatology, 28(10), 657-664. https://doi.org/10.1038/jp.2008.62

Coustan, D. R. (2013). Gestational Diabetes Mellitus. Clinical Chemistry, 59(9), 1310-1321. https://doi.org/10.1373/clinchem.2013.203331

Di Biase, N., Balducci, S., Lencioni, C., Bertolotto, A., Tumminia, A., Dodesini, A. R., Pintaudi, B., Marcone, T., Vitacolonna, E., \& Napoli, A. (2019). Review of general suggestions on physical activity to prevent and treat gestational and pre-existing diabetes during pregnancy and in postpartum. Nutrition, Metabolism and Cardiovascular Diseases, 29(2), 115-126. https://doi.org/10.1016/j.numecd.2018.10.013

Ferreira, L. A. de P., Piccinato, C. de A., Cordioli, E., \& Zlotni1, E. (2019). Índice De Massa Corporal Pré-Gestacional, Ganho De Peso Na Gestação E Resultado Perinatal: Estudo Descritivo Retrospectivo. Hospital Israelita Albert Einstein, São Paulo, SP, Brasil, 17(2), 1-16. https://doi.org/10.31744/einstein 
Research, Society and Development, v. 10, n. 8, e25610817062, 2021

(CC BY 4.0) | ISSN 2525-3409 | DOI: http://dx.doi.org/10.33448/rsd-v10i8.17062

Julia, A., Souza, D., Rocha, E., Morelli, F., Lyrio, L., \& Jardim, M. (2017). Diabetes Mellitus Gestacional E Pré-Gestacional. Revista Cadernos de Medicina, $02(3), 40-47$.

Koning, S. H., Hoogenberg, K., Lutgers, H. L., van den Berg, P. P., \& Wolffenbuttel, B. H. R. (2016). Gestational Diabetes Mellitus:current knowledge and unmet needs. Journal of Diabetes, 8(6), 770-781. https://doi.org/10.1111/1753-0407.12422

Lende, M., \& Rijhsinghani, A. (2020). Gestational Diabetes: Overview with Emphasis on Medical Management. International Journal of Environmental Research and Public Health, 17(24), 9573. https://doi.org/10.3390/ijerph17249573

Maganha, C. A., Abrão, K. C., \& Zugaib, M. (2004). Tratamento do diabetes melito gestacional baseado em achados ultra-sonográficos. Revista Da Associação Médica Brasileira, 50(3), 235-236. https://doi.org/10.1590/s0104-42302004000300009

Menicatti M., Fregonesi C. E. P. T. (2006) Diabetes Gestacional: Aspectos fisiopatológicos e tratamento. Arq. Ciênc. Saúde Unipar, 10(2): 105-111.

Pereira A. S. et al. (2018). Metodologia da pesquisa científica. UFSM.

Simon, C. Y., Marques, M. C. C., \& Farhat, H. L. (2013). Glicemia de jejum do primeiro trimestre e fatores de risco de gestantes com diagnóstico de diabetes melito gestacional. Revista Brasileira de Ginecologia e Obstetricia, 35(11), 511-515. https://doi.org/10.1590/S0100-72032013001100006

Sociedade Brasileira de Diabetes. (2015). Diabetes mellitus gestacional: diagnóstico, tratamento e acompanhamento pós-gestação. Diretrizes Da Sociedade Brasileira de Diabetes, 192-197.

Sociedade Brasileira de Diabetes. (2020). Diretrizes da Sociedade Brasileira de Diabetes 2019-2020.

Souza, M. L. R. De, Silva, R. R. E., Silva, T. R. E., Oliveira, L. C. De, Dienstmann, G., Nascimento, I. B. Do, \& Silva, J. C. (2019). Factors Associated with the Need for Insulin as a Complementary Treatment to Metformin in Gestational Diabetes Mellitus. Revista Brasileira de Ginecologia e Obstetricia, 41(12), 697702. https://doi.org/10.1055/s-0039-1700796

Spaight, C., Gross, J., Horsch, A., \& Puder, J. J. (2016). Gestational Diabetes Mellitus. Endocrine Development, 163-178. https://doi.org/10.1159/000439413

Zheng, J., Wang, H., \& Ren, M. (2017). Influence of exercise intervention on gestational diabetes mellitus: a systematic review and meta-analysis. Journal of Endocrinological Investigation, 40(10), 1027-1033. https://doi.org/10.1007/s40618-017-0673-3

Weinert, L. S., Silveiro, S. P., Oppermann, M. L., Salazar, C. C., Simionato, B. M., Siebeneichler, A., \& Reichelt, A. J. (2011). Diabetes gestacional: um algoritmo de tratamento multidisciplinar. Arquivos Brasileiros de Endocrinologia \& Metabologia, 55(7), 435-445. https://doi.org/10.1590/S000427302011000700002 